\title{
Simultaneous sampling and length contrast
}

\author{
KEVIN JORDAN and PETER W. ENGLISH \\ San Jose State University, San Jose, California
}

\begin{abstract}
The illusion of perceived line length produced by the parallel lines configuration reverses from assimilation to contrast with large spatial separation of the contextual and test lines (Jordan \& Schiano, 1986). In the present experiment, we tested whether length contrast is produced by the sequential sampling of contextual and test lines or by the simultaneous sampling of the stimuli from a spatially distributed attentive field. Scanning instructions were manipulated such that one group of observers was encouraged to sample the contextual and test lines simultaneously, a second group was encouraged to sample the lines sequentially, and a third group was given no specific scanning instructions. The data indicated that the reversal to length contrast is produced by simultaneous sampling of contextual and test lines. Implications for attentional descriptions of visual illusions are discussed.
\end{abstract}

Since first being proposed by Pressey and Murray (1976) as a basic paradigm for geometric illusions such as the Müller-Lyer, Baldwin, and Ponzo illusions, the "parallel lines" illusion has been used as a minimal configuration for producing distortions of perceived length (e.g. , Brigell \& Uhlarik, 1979; Jordan \& Schiano, 1986; Jordan \& Uhlarik, 1985; Schiano, 1986). In its usual form, a $90-\mathrm{mm}$ (for example) contextual line that is spatially proximal to a parallel $60-\mathrm{mm}$ test line will produce overestimation of the length of the test line. This distortion of the perceived length of a test line toward the contextual-line length is labeled length assimilation. Distortions of perceived test-line length away from the contextual length are labeled length contrast.

Only within the last few years, and within a restricted set of conditions, has it become clear that length contrast can be produced by the Baldwin (Pressey $\&$ Wilson, 1980), Müller-Lyer (Jordan \& Uhlarik, 1986), and parallel lines figures (Brigell \& Uhlarik, 1979; Jordan \& Schiano, 1986; Jordan \& Uhlarik, 1985). Given these demonstrations of bidirectional length distortions, it has become necessary to develop a unified model that can account for both assimilation and contrast. Most importantly, such a model should propose a set of conditions under which a shift from assimilation to contrast is realized.

Jordan and Schiano (1986; see also Jordan \& Haleblian, 1988) have proposed a descriptive model of length assimilation and contrast that was adapted from Girgus and Coren's (1982) "pool-and-store" model of length dis-

The authors would like to express their appreciation to Alexander Pressey, Christopher Tyler, and an anonymous reviewer for their comments on earlier versions of the manuscript. In particular, Professor Pressey was helpful with the specification-of-assimilation theory and the anonymous reviewer pointed out the need for discussing the effects of retinal eccentricity on length perception. Peter W. English is now at the University of Arizona. Requests for reprints may be sent to Kevin Jordan, Department of Psychology, San Jose State University, San Jose, CA 95192. tortion and Pressey's assimilation theory (e.g., Pressey \& Murray, 1976). According to Girgus and Coren, "the pool-and-store model simply proposes that the observer uses each single fixation or glance to garner a global impression of one part of the stimulus array" (p. 558). Thus, a single fixation provides a sample of stimulus information from a finite spatial region. Girgus and Coren defined the spatial region sampled as the part of the stimulus array that falls within the fovea during a fixation. When more than one stimulus is contained in the spatial region sampled (i.e., the fovea), such as when the contextual and test lines are spatially proximal, the model proposes that the representations of their lengths will be pooled, resulting in assimilation. Contrast results when the contextual and test lines are so spatially distal that the representations of their lengths cannot be sampled from the information contained in a single fixation. These conditions of extrafoveal separation of contextual and test lines necessitate multiple fixations, with the stored representation of one line's length contrasted to that of the other.

Jordan and Schiano (1986) agree that only a limited spatial region of the stimulus array can be sampled by a single fixation. However, their model differs from the Girgus and Coren (1982) model in the operational definition of the size of the spatial region sampled during a fixation. Jordan and Schiano's data led them to propose that the size of the sampled region is variable, rather than absolute, and is determined by the length of the test line. Here, they borrowed from Pressey's assimilation theory and argued that assimilation occurs when the contextual and test lines fall within an "attentive field" whose size is relationally determined (Pressey \& DiLollo, 1978). Specifically, Jordan and Schiano proposed that the radius of the attentive field is the test-line length; small test lines produce small attentive fields and large test lines produce large attentive fields. Experimental support for this proposal comes from studies that show that whenever a horizontal contextual line is separated from a parallel test 
line by more than the test-line length, a reversal from length assimilation to length contrast is observed (Jordan \& Haleblian, 1988; Jordan \& Schiano, 1986).

It is important to point out that the Jordan and Schiano (1986) model differs from Pressey and DiLollo's (1978) specification of the attentive field (see Pressey, 1988). According to Jordan and Schiano, the assimilation region is circular, centered at the center of the test line, and has a radius equal to the test-line length. Pressey and DiLollo also proposed that the assimilation region is circular, but they argued that the center of this region is the midpoint of the greatest distance between the test and comparison lines and has a radius determined by that distance. Thus, Pressey's assimilation theory takes the comparison stimulus (the measuring instrument) into account when specifying attentive field size (Pressey, 1987), whereas the Jordan and Schiano model does not. Jordan and English (1988) reported data supporting Jordan and Schiano's (1986; see also Jordan \& Haleblian, 1988) specification of the attentive field center and radius.

Implicit in the Jordan and Schiano (1986) and Jordan and Haleblian (1988) descriptions of length distortions is that the reversal of assimilation to contrast is mediated by a shift from simultaneous sampling (and hence pooling) of the contextual and test lines to sequential sampling of the two lines (cf. Girgus \& Coren, 1982). An alternative to this sequential sampling, or "dual glance," model of length contrast has been suggested by Jordan and Randall (1987) on the basis of the work of Pressey and Wilson (1980). According to Jordan and Randall, the attentive field might actually be composed of two spatially distinct regions, a central assimilation region and an annular contrast region (see Figure 1). They proposed that assimilation occurs when both the contextual and the test lines fall within the central region of the attentive field. Note that this assimilation region is centered on the test line and has a radius equal to the test-line length (Jordan \& English, 1988; see above). Length contrast results when the contextual line falls into the outer, annular region of the attentive field while the test line remains within the central region of the field. This notion of a bidirectional field was initially proposed by Pressey and Wilson in an attempt to account for length contrast in the Baldwin figure. However, they proposed that an interactive field, which "reflects spatial contour interaction effects" rather than attentional deployment (Pressey, 1988, p. 201) might be composed of an assimilation region surrounded by an annular contrast region. Pressey has not elaborated much on this initial suggestion, perhaps because he questions the reliability of length contrast (Pressey, 1988).

The major difference between the model of length assimilation and contrast as stated by Jordan and Schiano (1986) and by Jordan and Randall (1987) is in the description of length contrast. Whereas Jordan and Schiano proposed that sequential sampling of the lengths of contextual and test lines produces length contrast, Jordan and Randall proposed that contrast results from the simultaneous sampling of the lengths of contextual and test lines

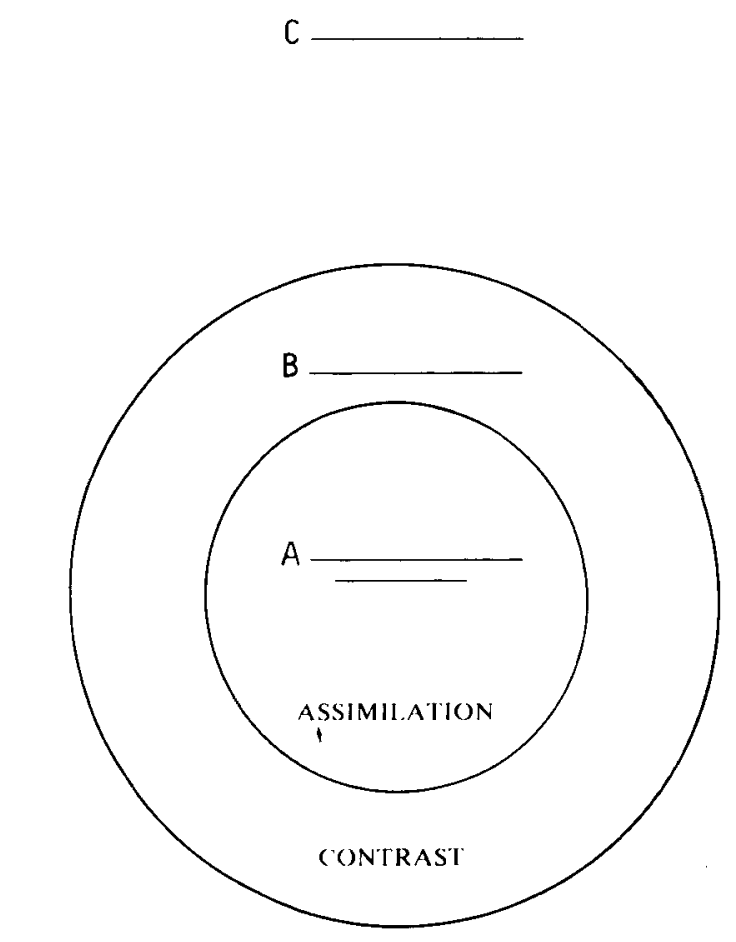

Figure 1. Schematic representation of the bidirectional attentive field proposed by Jordan and Randall (1987). This bidirectional field notion was adapted from Pressey and Wilson (1980) but difiers in significant ways from their proposal, as noted in the text. When both the contextual and the test lines are within the central region (A), assimilation occurs. When the contextual line falls within the outer region while the test line is in the central region (B), contrast occurs. Finally, when the contextual line is outside of the attentive field (C), there is no observed distortion of the perceived length of the test line in the center of the field. The assimilation region of the attentive field is centered at the center of the test line and has a radius equal to the test-line length (Jordan \& English, 1988).

from two spatially distinct regions of an attentive field. The purpose of the present experiment was to test between such sequential and simultaneous models of length contrast. We attempted to determine whether length contrast occurs in the parallel lines array due to the contextual line being outside the attentive field of the parallel test line (in which case sequential sampling is required) or whether it is due to simultaneous sampling of the lengths of the contextual and test lines from two spatially distinct regions of the attentive field.

Specifically, we manipulated sampling instructions to encourage observers to sample the lengths of the contextual and test lines either simultaneously or sequentially. One group of observers was given sequential sampling instructions in which they were told to inspect the contextual line first and then to inspect the test line and make a judgment. A second group was given simultaneous sampling instructions in which they were told to view only the test line; they were not to look at the contextual line. Thus, any effect of the contextual line on length judgments would be due to simultaneous sampling of the lengths of 
the contextual and test lines (assuming that the observers followed instructions). Finally, since we gave no explicit instructions regarding sampling of the lengths of contextual and test lines in previous studies demonstrating length contrast in the parallel lines array (Jordan \& Haleblian, 1988; Jordan \& Schiano, 1986), we included a condition in which no instructions regarding scanning were given. This condition allowed observers to sample the lengths of the two lines according to their own biases and served as a useful baseline against which to compare the other two conditions.

In addition to the manipulation of scanning instructions, we manipulated the vertical spatial separation of the horizontal contextual line from the parallel test line. The purpose of this manipulation was to determine whether the shift from length assimilation (under conditions of small spatial separation) to length contrast (under conditions of large spatial separation) could be influenced by whether observers sampled the lengths of the contextual and test lines simultaneously or sequentially.

If the shift from length assimilation to contrast in the sequential sampling condition is similar to the baseline, no-instructions condition, then the Jordan and Schiano (1986) proposal of a unidirectional attentive field in which assimilation occurs would be supported. However, if the shift from assimilation to contrast in the simultaneous sampling condition most resembles the baseline, noinstructions condition, then the Jordan and Randall (1987) model of a bidirectional attentive field in which both assimilation and contrast can occur (depending on spatial location within the field) would be supported.

\section{METHOD}

\section{Observers}

Fifty-four members of an introductory psychology course at San Jose State University participated in the experiment in order to earn course credit. All observers were run in individual sessions.

\section{Stimuli and Design}

All stimulus lines were made with black tape on a white background. They were then photographed and the resulting $35-\mathrm{mm}$ slides were projected on the rear of a translucent screen. Order and duration of stimulus presentation were controlled by a programmable Bell \& Howell random-access projector (Model 850A).

The lengths of the contextual and test lines were chosen to maximize length assimilation and contrast prior to the manipulation of scanning instructions. Brigell and Uhlarik (1979) and Schiano (1986) reported that length assimilation is greatest when the ratio of contextual length to test length (the "framing ratio") is between 1.5 and 2.0. In addition, Brigell and Uhlarik reported that length contrast peaks when the framing ratio is 0.67 . On the basis of these findings, the projected contextual lengths used in the present experiment were 90 and $40 \mathrm{~mm}$ and the test length was $60 \mathrm{~mm}$, resulting in framing ratios of 1.5 and 0.67 , respectively. The thickness of the lines on the viewing screen was $1.5 \mathrm{~mm}$ and the viewing distance was $60 \mathrm{~cm}$.

The horizontal, $60-\mathrm{mm}$ test line was centered on the viewing screen and the parallel contextual line was centered above it at one of three vertical spatial separations: 5,100 , or $300 \mathrm{~mm}$. According to both the Jordan and Schiano (1986) and the Jordan and Randall (1987) models, length assimilation should occur in the $5-\mathrm{mm}$ condition since both the contextual line and the test line would be within the region that would produce assimilation, and length contrast should occur in the 100-mm condition since the contextual line would be outside the assimilation region. On the basis of estimates of the boundaries of the contrast region of the attentive field made by Jordan and Randall, no distortion of perceived test-line length would be expected in the $300-\mathrm{mm}$ condition. The important consideration for the present experiment was whether the shift from length assimilation to length contrast was affected by scanning instructions as the spatial separation of the contextual and test lines increased from 5 to $100 \mathrm{~mm}$.

To summarize the design of the experimental slides, there were two levels of contextual line length $(40$ and $90 \mathrm{~mm})$ crossed with three levels of spatial separation $(5,100$, and $300 \mathrm{~mm})$, for a total of six slides.

Judgments of test-line length were obtained using a graded-series comparison scale (see Coren \& Girgus, 1972a), which was located $15 \mathrm{~cm}$ to the right of the midpoint of the test line on each slide. The scale consisted of 11 horizontally oriented lines, labeled A through $\mathrm{K}$, which ranged in length from 50 to $70 \mathrm{~mm}$ in 2-mm increments. The point of objective equality (POE) on this scale was $F$, or $60 \mathrm{~mm}$ (i.e., the scale length that physically matched the test length). The lines on the scale were $1.5 \mathrm{~mm}$ thick and were separated vertically by $10 \mathrm{~mm}$. To prevent response bias, we encouraged the observers to use a wide range of the response scale by including three filler stimuli in the design. The test lengths for these stimuli ranged from 52 to $68 \mathrm{~mm}$. Responses to the filler stimuli were not included in the data analysis.

Finally, to familiarize the observers with the use of the response scale, there were six practice stimuli, which consisted of only a test line (no contextual line). The test lengths of these stimuli were $52,56,60,60,64$, and $68 \mathrm{~mm}$.

\section{Procedure}

The observers viewed the projected image of the stimuli under normal fluorescent room lighting. The observers were familiarized with the range and variety of the stimuli and with the use of the response scale. Specifically, they were told that the task was to match the apparent length of the test line to one of the 11 lines on the response scale by verbally reporting the letter of the match to the experimenter.

Eighteen observers were assigned to each of the three instructional conditions. The observers in the simultaneous sampling condition were read the following instructions:

Often, when we present a slide with two lines, people have their attention drawn to the upper, nonjudged line. I don't want you to do that. In fact, I will be even more explicit. When the stimulus slide appears, ignore the upper line and look only at the lower line and make your length match of the lower line to one of the lines on the graded-series scale to the right.

If the observers followed instructions, any influence of the upper, contextual line would be incidental to, and simultaneous with, the sampling of the length of the test line. The observers in the sequential sampling condition were read the following instructions:

Often, when we present a slide with two lines, people attempt to ignore the upper, nonjudged line. I don't want you to do that. In fact, I will be even more explicit. When the stimulus slide appears, look first at the upper line and then look at the lower line and make your length match of the lower line to one of the lines on the graded-series scale to the right.

The observers in the baseline condition were not given any specialized instructions regarding sampling of the lengths of the contextual and test lines.

After these instructions, the observers proceeded through a random order of the six practice stimuli, which was followed by two random orders of the six test and three filler stimuli. A trial con- 
Table 1

Observers' Self-Reports of Sampling Strategies

\begin{tabular}{|c|c|c|c|}
\hline \multirow[b]{2}{*}{ Question } & \multicolumn{3}{|c|}{ Sampling Condition } \\
\hline & Simultaneous & Sequential & Baseline \\
\hline \multicolumn{4}{|l|}{$\begin{array}{l}\text { When both lines were } \\
\text { present, did you look } \\
\text { at the upper line? }\end{array}$} \\
\hline Never & 4 & 0 & 3 \\
\hline Sometimes & 14 & 3 & 13 \\
\hline Always & 0 & 15 & 2 \\
\hline \multicolumn{4}{|l|}{$\begin{array}{l}\text { If sometimes, were you } \\
\text { more likely to look at } \\
\text { the upper line when the } \\
\text { lines were }\end{array}$} \\
\hline Close $(5 \mathrm{~mm})$ & 12 & 3 & 12 \\
\hline Medium (100 mm) & 2 & 0 & 0 \\
\hline Far $(300 \mathrm{~mm})$ & 0 & 0 & 1 \\
\hline
\end{tabular}

Note $-N=18$ in each condition.

sisted of a 5-sec presentation of the test or filler slide during which the length judgment was to be made. The slide contained the contextual and test lines as well as the graded-series comparison scale in the spatial locations described above. Trials were separated by $10 \mathrm{sec}$, during which the viewing screen was blank. In all, each of the 54 observers made 24 length judgments: six practice judgments and two judgments each of the six test and three filler stimuli. The entire procedure required approximately $10 \mathrm{~min}$ for each observer.

Finally, to assess the observers' strategies for sampling the lengths of the contextual and test stimuli, all observers responded to two questions subsequent to the length judgments. These questions, as well as the response alternatives and the observers' responses, are presented in Table 1.

\section{RESULTS AND DISCUSSION}

Table 1 presents the responses of the observers in each of the three instructional conditions to the two questions regarding scanning strategies. In general, the responses of the observers indicate that they followed instructions, although observers in the simultaneous sampling group did report looking at the contextual line when it was $5 \mathrm{~mm}$ above the test line. This is not surprising given the proximity of the contextual and test lines (at the $60-\mathrm{cm}$ viewing distance, the two lines were separated by less than $0.5^{\circ}$ ). Most importantly, observers in the baseline condition reported a scanning strategy quite similar to that of the simultaneous sampling group; they too reported looking at the contextual line at the 5-mm spatial separation from the test line but not at the 100 - or $300-\mathrm{mm}$ separations.

The responses to the $60-\mathrm{mm}$, no-context lines obtained during the practice series were used to estimate the point of subjective equality (PSE) of the $60-\mathrm{mm}$ line contained in the six test stimuli for each group of observers. The means of the 36 responses to this control line (two responses from each of the 18 observers in the group) were $56.5,56.8$, and $55.8 \mathrm{~mm}$ for the baseline, simultaneous sampling, and sequential sampling conditions, respectively. Such an underestimation of the objective length of the $60-\mathrm{mm}$ test line has been reported consis- tently in studies using the graded-series comparison scale (Brigell \& Uhlarik, 1979; Jordan \& Haleblian, 1988; Jordan \& Schiano, 1986; Jordan \& Uhlarik, 1985, 1986).

Within each instructional condition, the observers' length judgments for the 60-mm test line contained in each of the six test slides were converted into a measure of illusion magnitude (mean deviation from PSE) by subtracting the PSE for that condition from the judged length of the test line . These data are presented in Figures $2 \mathrm{~A}-2 \mathrm{C}$. First, note the substantial assimilation produced by spatial proximity of the contextual and test lines (see the curves labeled " $5 \mathrm{~mm}$ " in Figures $2 \mathrm{~A}-2 \mathrm{C}$ ). For all three instructional conditions, the $90-\mathrm{mm}$ contextual line produced overestimation of the 60 -mm test line, whereas the $40-\mathrm{mm}$ contextual line produced either no distortion or some slight underestimation of the $60-\mathrm{mm}$ test line. Thus, assimilation was observed in the sequential sampling condition at the 5-mm spatial separation even though the observers were to sample the contextual and test stimuli sequentially. A'ssuming that the observers were able to sample the two stimuli sequentially (which is what they reported; see Table 1), the finding of assimilation indicates that dual glances, per se, are not the stimulus to a reversal to length contrast.

In the context of the Jordan and Schiano (1986) and Girgus and Coren (1982) models of assimilation and contrast, it seems that eye movements do not control whether assimilation or contrast occurs. In the 5-mm spatial separation conditions, the contextual and test lines fall within the center of the attentive field (see Figure 1). It seems that it is the boundary of this assimilation region, rather than some behavioral manipulation, that determines the reversal to contrast.

At the 100 -mm spatial separation, a reversal to length contrast was observed for the baseline and simultaneous sampling conditions, but not for the sequential sampling condition (see curves labeled $100 \mathrm{~mm}$ in Figures $2 \mathrm{~A}-2 \mathrm{C}$ ). The 40-mm contextual line now produced overestimation of the $60-\mathrm{mm}$ test line, whereas the 90 -mm contextual line produced underestimation or no distortion. This reversal to contrast in the baseline, no-instructions condition replicates Jordan and Schiano's (1986) original report of length contrast in the parallel lines array under conditions of spatial separation (see also Jordan \& Haleblian, 1988, Experiment 3). Given the observers' reports of their sampling strategies (see Table 1), these data indicate that the observers simultaneously sampled the contextual and test elements despite a $100-\mathrm{mm}$ spatial separation. Thus, simultaneous sampling does not always lead to assimilation as Girgus and Coren (1982) proposed. On the other hand, there was no length contrast at the $100-\mathrm{mm}$ spatial separation of contextual and test lines for the sequential sampling condition. As with the 5-mm spatial separation data, the failure of sequential sampling to produce length contrast indicates that dual glances, per se, do not produce length contrast. Rather, it seems that the relative location of the contextual and test lines within a bidirectional attentive field, as proposed by Jordan and Randall (1987), 
A. SIMULTANEOUS

$\mathrm{O}-\mathrm{O} 5 \mathrm{~mm}$

$\triangle \longrightarrow \quad \triangle 100 \mathrm{~mm}$

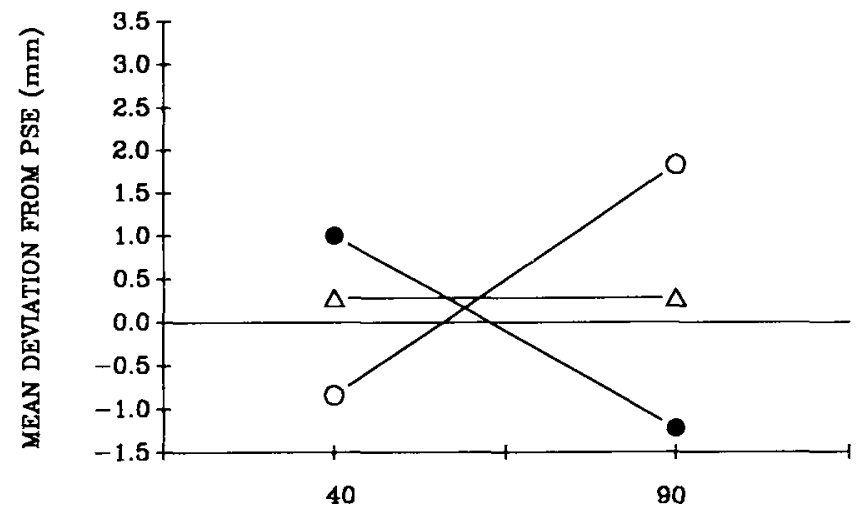

CONTEXTUAL LINE LENGTH (mm)

B. SEQUENTIAL

$$
\begin{aligned}
& O-05 \mathrm{~mm} \\
& -\triangle 100 \mathrm{~mm} \\
& \triangle-\triangle 300 \mathrm{~mm}
\end{aligned}
$$

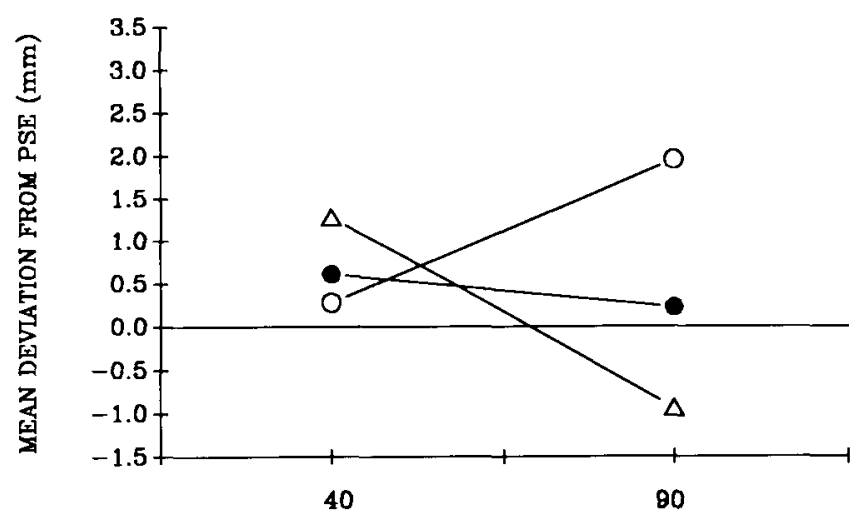

CONTEXTUAL LINE LENGTH (mm)

C. BASELINE

$\mathrm{O}-\mathrm{O} 5 \mathrm{~mm}$

$100 \mathrm{~mm}$

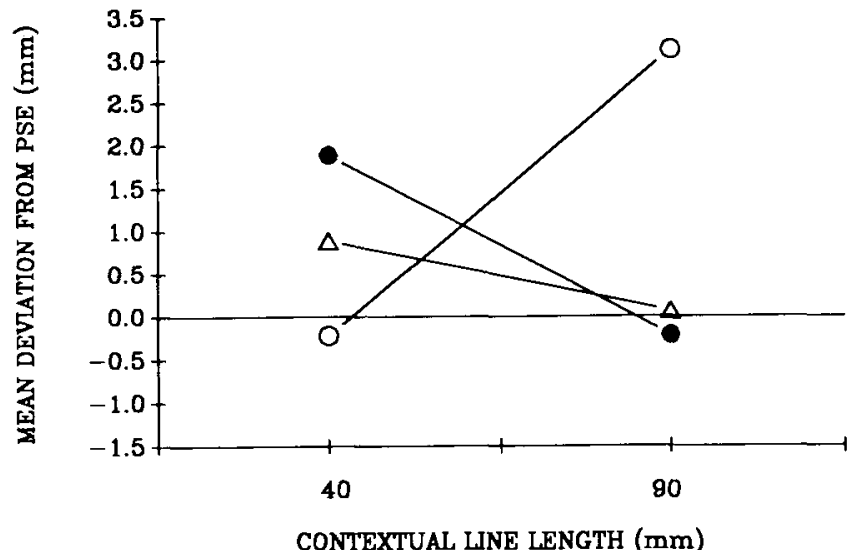

CONTEXTUAL LINE LENGTH (mm)

Figure 2. Mean deviations from the point of subjective equality (PSE) as a function of contextual-line length and spatial separation for the (A) simultaneous, (B) sequential, and (C) baseline conditions. 
determines whether length assimilation or contrast is observed.

Finally, at the $300-\mathrm{mm}$ spatial separation, length contrast was observed only in the sequential sampling condition. No length contrast was anticipated in the baseline or simultaneous sampling conditions with the $300-\mathrm{mm}$ separation. This prediction was based on Jordan and Randall's (1987) estimate that the outer boundary of the contrast region of the bidirectional attentive field is 2.5 testline lengths from the center of the field (at the $300-\mathrm{mm}$ separation in the present experiment, the contextual line was 5 test-line lengths from the center of the $60-\mathrm{mm}$ test line). Taken together with the results at the 5- and 100$\mathrm{mm}$ levels of spatial separation for the sequential sampling conditions, the finding of length contrast at the 300 mm spatial separation indicates that dual glances can produce contrast, but only when the contextual line is outside the attentive field of the test line. This is consistent with the observation of length contrast within an aftereffect paradigm in which the contextual line is inspected prior to judging the test line (Brigell \& Uhlarik, 1979; Jordan \& Uhlarik, 1985).

The overall pattern of length distortions in Figures $2 \mathrm{~A}-2 \mathrm{C}$ and the self-reports of scanning strategies in Table 1 indicate a greater similarity of the baseline condition to the simultaneous sampling condition than to the sequential sampling condition. Assuming that similar data imply similar processes, the data support Jordan and Randall's (1987) proposal of a spatially distributed attentive field in which two types of qualitatively different processing occur (see Figure 1). This conclusion was assessed statistically. The deviations from PSE were analyzed in a 3 (instructional conditions) $\times 3$ (spatial separations) $\times$ 2 (contextual lengths) $\times 2$ (replications) $\times 18$ (observers) mixed analysis of variance (ANOVA). Instructional condition was a between-subjects variable. In this analysis, the three-way interaction of instructional condition, spatial separation, and contextual length was statistically significant $[F(4,102)=4.59, p<.01]$, as were the twoway interaction of spatial separation and contextual length $[F(2,102)=65.96, p<.01]$ and the main effect of spatial separation $[F(2,102)=8.99, p<.01]$. No other main effects or interactions reached statistical significance in the analysis.

The spatial separation $x$ contextual length interaction reflects the reversal from length assimilation to length contrast with increasing spatial separation of the contextual and test lines; that is, long contextual lines produced overestimation at the 5-mm separation, but short contextual lines produced overestimation at larger spatial separations. The three-way interaction indicates that the pattern of this reversal varied with instructional condition. Since the primary purpose of the present experiment, as stated in the introduction, was to determine whether the simultaneous or the sequential sampling condition produced effects most similar to the baseline condition, the three-way interaction was examined further. Two separate 2 (instructional conditions) $\times 3$ (spatial separations) $\times 2$ (contextual lengths) $\times 2$ (replications) $\times 18$ (observers) mixed
ANOVAs were done. In both analyses, instructional condition was a between-subjects variable.

The first analysis compared the sequential sampling condition to the baseline, no-instruction condition. In this analysis, the three-way interaction of instructional condition, spatial separation, and contextual length was significant $[F(2,68)=4.8, p=.011]$, as were the interaction of spatial separation and contextual length $[F(2,68)$ $=38.74, p<.01]$ and the main effect of spatial separation $[F(2,68)=8.26, p<.01]$. No other main effects or interactions reached significance in the analysis. Again, as with the overall analysis, the interaction of spatial separation and contextual length indicates the reversal from length assimilation to length contrast. However, the three-way interaction indicates that the pattern of the reversal was different for the sequential and baseline conditions.

The second analysis compared the simultaneous sam" pling condition to the baseline condition. The two-way interaction of spatial separation and contextual length reached statistical significance $[F(2,68)=58.33, p<$ $.01]$, as did the main effect of spatial separation $[F(2,68)$ $=4.72, p=.012$ ]. No other main effects or interactions were significant. Again, the interaction of spatial separation and contextual length indicates the reversal from length assimilation to length contrast. The lack of any interaction involving instructional condition or a main effect of instructional condition indicates that the pattern of length distortions was statistically indistinguishable in the baseline and simultaneous sampling conditions. Thus, the graphical and statistical analyses and the self-reports of the observers' sampling strategies indicate similar processing of contextual and test lines in the parallel lines array for the baseline and simultaneous sampling conditions.

\section{Bidirectional Attentive Fields and Length Illusions}

The results of the present experiment fail to support Jordan and Schiano's (1986) revision of Girgus and Coren's (1982) pool-and-store model of length assimilation and contrast. Central to the pool-and-store model is the assertion that the information-processing system imposes constraints on the amount of information that can be sampled in a single glance, and that these sampling limitations produce various perceptual distortions (Girgus \& Coren, 1982). According to this model, when contextual and test stimuli are sampled in a single glance, the representations of their lengths are "pooled," resulting in assimilation. On the other hand, any manipulation that necessitates sampling the contextual and test stimuli by more than a single glance was proposed to produce "storing" of the representation of contextual length that contrasted with the representation of test-line length. Two key findings of the present experiment point to the shortcomings of this model. First, simultaneous sampling does not always lead to assimilation as the pool part of the model would suggest. Thus, the observation of length contrast under simultaneous sampling of contextual and test lines at the 
100-mm spatial separation is inconsistent with Jordan and Schiano's revision of the pool-and-store model. Second, sequential sampling does not always lead to length contrast as the store part of the model requires. For example, length assimilation was observed under sequential sampling instructions at the 5-mm spatial separation, and no distortion was observed with sequential sampling at the 100 -mm separation.

While the data point out the shortcomings of the pooland-store model, they are supportive of the bidirectional field model first suggested by Pressey and Wilson (1980) and later modified by Jordan and Randall (1987). Pressey and Wilson proposed that the bidirectional field is an interactive, rather than an attentive, field (see Pressey, 1988), whereas Jordan and Randall's (1987) and Jordan and English's (1988) data led them to propose that the attentive field is partitioned into two spatially distinct regions (see Figure 1). The essential feature of the model is that there is a spatial distribution of attention across two regions that process stimuli in a qualitatively distinct manner. Thus, when the contextual and test stimuli are within the central region of the attentive field (see $A$ in Figure 1), assimilation results. In addition, when the contextual line falls within the annular region of the attentive field while the test line is within the central region (see B in Figure 1), contrast results. Finally, when the contextual line is outside the attentive field (see $C$ in Figure 1), there is no distortion of the test line in the central region of the field.

As the model in Figure 1 is applied to geometric visual illusions, it demonstrates great power in describing these phenomena. However, there are several tasks ahead in the development of this bidirectional attentive field model to describe visual illusions. First, parametric investigations of the shape and boundaries of the assimilation and contrast regions of the attentive field seem necessary. Second, it is important to determine whether the model generalizes beyond illusions of linear extent to illusions of area such as the Ebbinghaus and Delboeuf illusions.

\section{Geometric Illusions and Attention}

The use of attention as a construct mediating illusions is not a new idea. For example, shifts of attention, or centrations, were the central theme of Piaget's (1969) approach to visual illusions. However, the use of attention in the present model as a construct mediating length assimilation and contrast might allow theories of illusions to more clearly cortribute to theories of perception. As Zusne (1986) has pointed out, the literature on visual illusions is large, with more than 1,100 articles published from the mid-19th century to 1986 and more than 100 articles published during each 5-year period from 1965 to 1984. Perhaps unfortunately, this large body of literature also seems to be free-standing, which raises the possibility that theories of illusions may have little to say about normal perception. An elaboration of the role of attention in illusions might allow for the exciting possibility that this large, seemingly free-standing body of liter- ature on visual illusions could contribute to our understanding of visual spatial attention. Conversely, the literature on visual attention as revealed through the visual-search, or target-detection, paradigm (e.g., Eriksen \& St. James, 1986) might be expected to contribute to our understanding of illusions.

As a first step in exploring the role of attention in illusions, it can be noted that if the present model is correct in proposing that the spatial distribution of attention mediates illusions of perceived line length, any experimental manipulation affecting the deployment of attention should affect the nature and the magnitude of the illusions (cf. Coren \& Girgus, 1978, p. 177). This seems to be the case. For example, Coren and Girgus (1972b) reported a change in the magnitude of the Müller-Lyer illusion when observers were instructed to attend to the test line and not to the contextual "wings." Additional support for the role of attention comes from the present experiment. We demonstrated a change in the nature of the illusion at both the 100 - and the $300-\mathrm{mm}$ spatial separations with a change in attentional allocation. Thus, length contrast was observed at the $100-\mathrm{mm}$ spatial separation of contextual and test lines in the simultaneous sampling condition but not in the sequential sampling condition (compare the 100mm curves in Figures $2 \mathrm{~A}$ and 2B). The observers' reports of sampling strategies indicated that they did not move their eyes in the simultaneous sampling condition and that they did move their eyes in the sequential sampling condition. Since eye movements do not occur without a corresponding shift in attention (Shepard, Findlay, \& Hockey, 1986), it is reasonable to conclude that a shift in the allocation of attention mediated the differences between the observed illusion for the simultaneous and sequential sampling conditions.

The model of the attentive field we propose is that there is a variable-size field within which parallel processing of features such as length occurs. The proposal of parallel processing of length is consistent with our conclusion that length assimilation and contrast result from the simultaneous processing of test and contextual lines. There exists a large, current body of literature on visual processing in which search tasks have been used to investigate the nature of feature analysis in early vision (e.g., Treisman \& Souther, 1985). According to Treisman and Gormican (1988), "most theorists agree that early description derives from spatial groupings of a small set of simple primitives that are registered in parallel across the visual field" (p. 15). A partial listing of these primitive features includes stimulus size, orientation, length, and color. Although a review of this literature is beyond the scope of the present paper, one theoretical point that seems directly relevant to length illusions concerns the output from parallel retrieval of primitive features contained in a stimulus array (i.e., retrieval without focused attention). Treisman and Souther $(1985$, p. 307$)$ argued that early vision is able to extract information concerning the presence of a feature in parallel and that this process could involve either a categorical signal of the presence or ab- 
sence of the feature or a graded signal proportional to the degree of presence of the feature throughout a stimulus array. If the latter is the case, the distortions we report may be a by-product of early visual processes that produce a pooled response to length across spatial locations.

Despite obvious differences in paradigms used in research on visual spatial attention (e.g., visual search) and visual illusions (e.g., size judgment), a link of the illusion literature to the attention literature seems justifiable, and perhaps fruitful, given the similarities of the issues addressed and the models proposed. Two similarities in particular stand out. First, several experiments on the spatial distribution of visual attention indicate that the size of the attentive field is variable and may depend on the specific task or the stimuli (e.g., Eriksen \& St. James, 1986; LaBerge, 1983). For example, LaBerge (1983) required observers to categorize five-letter words or the middle letter of five-letter words (or nonwords) prior to a secondary task of detecting the target digit 7 in one of the five spatial locations previously occupied by the letters. The time to detect the 7 in the secondary task did not depend on its spatial location subsequent to the word categorization. However, detection time increased in proportion with the distance from the middle spatial location in the letter-categorization conditions (both word and nonword). LaBerge interpreted these data to indicate that the visual attentive field width changes depending on the task. A popular metaphor used to describe the visual attentive field is a "spotlight" (but see Eriksen \& St. James, 1986, and Yantis, 1988). Classifying words produces a wider spotlight than does classifying letters. Simply, the size of the spotlight or attentive field is not absolute; it may be determined relative to the stimuli such that small focal stimuli produce small attentive fields and large stimuli produce large attentive fields.

In the illusion literature, this same conclusion of a variable-size attentive field has been reached by Jordan and Schiano (1986) and Pressey and DiLollo (1978). Jordan and Schiano reported that length assimilation in the parallel lines configuration reversed to length contrast under conditions of large spatial separation of contextual and test lines. Moreover, a larger spatial separation was needed to induce this reversal as test-line length increased. Borrowing Pressey's (1972) attentive field construct, Jordan and Schiano argued that small test lines produce small attentive fields and large test lines produce large attentive fields.

A second, and related, similarity of the two bodies of literature concerns the deployment of attention within an attentive field. A robust finding within the attention literature is a "distance effect." Referring to the LaBerge (1983) study described above, observers are often required to process a stimulus at one location (e.g., classifying the middle letter of a five-letter string) prior to processing a stimulus at a second spatial location (e.g., the digit 7 at the leftmost of five locations). As the distance between the two locations increases, the time necessary to detect the digit increases. This finding can be interpreted as reflecting the time for the metaphorical spotlight to "move" from one spatial location to another (but see Eriksen \& Murphy, 1987, and Yantis, 1988, for a critique of this conclusion), or it could reflect a gradient of processing across the attentive field (see LaBerge \& Brown, 1986, 1989).

Again, in the illusion literature, Jordan and Schiano (1986) reported a decreased effectiveness of the contextual stimulus in producing assimilation as the spatial separation of the stimuli increased. There appears to be a gradient of processing within the assimilation region of the attentive field. The adjacency postulate of assimilation theory is consistent with the idea of a processing gradient that influences illusion magnitude. This postulate states that the "effectiveness of a contextual magnitude decreases as the distance between the contextual and the focal (standard) magnitude increases" (Pressey \& Murray, 1976, p. 538). Accordingly, the focal or test stimulus receives the bulk of the attentional resources and the observed length distortions are a weighted average of the contextual and test lengths (see Brigell, Uhlarik, \& Goldhorn, 1977).

In summary, the present experiment indicates that simultaneous processing of contextual and test lines within a spatially distributed attentive field produces both assimilation and contrast of perceived line length. Which effect occurs depends upon the size of the central assimilation field as determined by the test-line length and the spatial separation of the contextual and test lines. The observation that illusion magnitude and direction depend on the size of the attentive field as determined by target size and the distance separating the contextual and test stimuli is consistent with many findings in the attention literature. Hopefully, by adjusting the temporal parameters of stimulus presentation in illusion experiments to approximate those used in attention research, we can be much more explicit about the role of attention in visual illusions.

\section{Attention or Retinal Eccentricity?}

Although we claim that the length distortions we study are the result of attentional factors, we must acknowledge the possibility that these distortions could also result from differences in length processing across retinal positions. For example, Schneider, Ehrlich, Stein, Flaum, and Mangel (1978) reported decreases in apparent line length as a line was moved from the center of a display to the periphery. Specifically, they found that apparent length is a power function of physical length, with the exponent depending on retinal position. There are several reasons for us to argue that our results are not due to retinal eccentricity. First, it is unclear whether Schneider et al.'s results, which were obtained under reduced-cue, monocular viewing conditions, would generalize to an illusion paradigm in which strong contextual factors operate. $\mathrm{Sec}_{-}$ ond, although apparent length clearly does vary with retinal position (Schneider et al., 1978), only the contextual line in our experiment would be affected by this variation, since the observers in both the simultaneous and the 
sequential instruction conditions were required to fixate the test line while making their length judgment. Third, the most important condition of the present experiment was probably the $100-\mathrm{mm}$ spatial separation, since it is under this condition that we have reliably demonstrated a reversal of length assimilation to contrast (Jordan \& Haleblian, 1988; Jordan \& Schiano, 1986). Given the 60$\mathrm{cm}$ viewing distance in the present experiment and fixation on the horizontal test line, the parallel contextual line was less than $10^{\circ}$ superior to the test line. However, Schneider et al.'s results (Experiment 4) indicate virtually no distortion of apparent length at this level of eccentricity. Combining the two points raised above, there is variation in apparent length as a function of retinal position, but this variation would only affect apparent contextual line length, and to such a minor extent that it could not account for the magnitude of the test-line length distortions we report. Finally, and most importantly, there are data that allow us to disentangle retinal sensitivity factors from attentional factors. For example, it is clear that the absolute spatial separation of contextual and test lines in the parallel lines figure is not important in determining whether length assimilation or length contrast is observed (Jordan \& Schiano, 1986; see also the present introduction and above). Rather, the spatial separation of the contextual and test lines, relative to the test-line length, determines the direction of the effect. Thus, it is possible to demonstrate length assimilation or length contrast with the same spatial separation and hence the same retinal eccentricity of the contextual line (Jordan \& Schiano, 1986). Conversely, Jordan and Schiano also demonstrated that the same magnitude of distortion (length assimilation, for example) can be observed at very different eccentricities. We argue that the test-line length determines the boundaries of an attentive field within which these distortions occur and that the position of the contextual line within this field, rather than its retinal location, determines whether length assimilation or length contrast is observed. We should point out that a very similar line of reasoning was used by LaBerge and Brown (1986, p. 189) to argue that the "attentional range effect" was due primarily to attentional factors rather than to retinal sensitivity.

\section{REFERENCES}

Brigell, M., \& UhLaRIK, J. (1979). The relational determination of length illusions and aftereffects. Perception, 8, 187-197.

Brigell, M., Uhlarik, J., GoldhorN, P. (1977). Contextual influences on judgments of linear extent. Joumal of Experimental Psychology: Human Perception \& Performance, 3, 105-118.

Coren, S., Girgus, J. S. (1972a). A comparison of five methods of illusion measurement. Behavior Research Methods \& Instrumentation, 4, 240-244.

Coren, S., GiRgus, J. S. (1972b). Differentiation and decrement in the Mueller-Lyer illusion. Perception \& Psychophysics, 12, 466-470.

Coren, S., \& Girgus, J. S. (1978). Seeing is deceiving: The psychology of visual illusions. Hillsdale, NJ: Eribaum.

ERIKSEN, C. W., MURPHY, T. D. (1987). Movement of attentional focus across the visual field: A critical look at the evidence. Perception \& Psychophysics, 42, 299-305.
ERIKSEN, C. W., \& ST. JAmes, J. D. (1986). Visual attention within and around the field of focal attention: A zoom lens model. Perception \& Psychophysics, 40, 225-240.

Girgus, J. S., CoREN, S. (1982). Assimilation and contrast illusions: Differences in plasticity. Perception \& Psychophysics, 32, 555-561.

JoRDAN, K., \& ENGUSH, P. W. (1988, November). Visual attentive fields and geometric illusions: Spatial characteristics. Paper presented at the annual meeting of the Psychonomic Society, Chicago.

JORDAN, K., \& HALEBLIAN, J. (1988). Orientation specificity of length assimilation and contrast. Perception \& Psychophysics, 43, 446-456.

JoRDAN, K., \& RANDALL, J. (1987). The effects of framing ratio and oblique length on Ponzo illusion magnitude. Perception \& Psychophysics, 41, 435-439.

JoRDAN, K., SCHIANO, D. J. (1986). Serial processing and the parallellines illusion: Length contrast through relative spatial separation of contours. Perception \& Psychophysics, 40, 384-390.

JoRDAN, K., \&HLARIK, J. (1985). Assimilation and contrast of perceived length depend on temporal factors. Perception \& Psychophysics, 37, 447-454.

JORDAN, K., \& UHLARIK, J. (1986). Length contrast in the Müller-Lyer figure: Functional equivalence of temporal and spatial separation. Perception \& Psychophysics, 39, 267-274.

LABERGE, D. (1983). Spatial extent of attention to letters and words. Journal of Experimental Psychology: Human Perception \& Performance, 9, 371-379.

LABERGE, D., \& BRown, V. (1986). Variations in size of the visual field in which targets are presented: An attentional range effect. Perception \& Psychophysics, 40, 188-200.

LABERGE, D., \& BRown, V. (1989). Theory of attentional operations in shape identification. Psychological Review, 96, 101-124.

Piaget, J. (1969). The mechanisms of perception (G. N. Seagrine, Trans.). New York: Basic Books.

Pressey, A. W. (1972). The assimilation theory of geometric illusions: An additional postulate. Perception \& Psychophysics, 11, 28-30.

Pressey, A. W. (1987). Psychophysical methods and life-span changes in visual illusion. Perceptual \& Motor Skills, 65, 83-87.

Pressey, A. W. (1988). A comment on "The effects of framing ratio and oblique length on Ponzo illusion magnitude." Perception \& Psychophysics, 43, 201-202.

Pressey, A. W., \& DiLollo, V. (1978). Effects of distance between standard and comparison lines on the Müller-Lyer illusion. Perception \& Psychophysics, 24, 415-419.

Pressey, A. W., Murray, R. (1976). Further developments in the assimilation theory of geometric illusions: The adjacency principle. Perception \& Psychophysics, 19, 536-544.

Pressey, A. W., Wilson, A. E. (1980). Assimilation theory and the Baldwin illusion. Italian Journal of Psychology, 7, 65-73.

SCHIANO, D. J. (1986). Relative size and spatial separation: Effects on the parallel-lines illusion. Perceptual \& Motor Skills, 63, 1151-1155.

Schneider, B., Ehrlich, D. J., Stein, R., Flaum, M., \& Mangel, S. (1978). Changes in the apparent lengths of lines as a function of degree of retinal eccentricity. Perception, 7, 215-223.

SHEPHERD, M., Findlay, J. M., \& HockeY, R. J. (1986). The relationship between eye movements and spatial attention. Quarterly Journal of Experimental Psychology, 38A, 475-491.

Treisman, A., \& Gormican, S. (1988). Feature analysis in early vision: Evidence from search asymmetries. Psychological Review, 95 , 15-48.

Treisman, A., \& Souther, J. (1985). Search asymmetry: A diagnostic for preattentive processing of separable features. Journal of Experimental Psychology: General, 114, 285-310.

Yantis, S. (1988). On analog movements of visual attention. Perception \& Psychophysics, 43, 203-206.

ZuSNE, L. (1986). Geometric-optical illusions: An update on the output of publications. Perceptual \& Motor Skills, 62, 729-730.

(Manuscript received January 25, 1988; revision accepted for publication May 23, 1989.) 